ÉLVIO TOGNOTTI'

FÁ́bIO RoBerto CABAR ${ }^{2}$

Leopoldo de Olivelra Tso ${ }^{3}$

JONATHAS BORGES SOARES ${ }^{4}$

Newton EDUARDo Busso ${ }^{5}$

NélSON ANTUNES JUNIOR ${ }^{6}$

Artigos originais

Palavras-chaves

Leuprolida/administração \& dosagem

Fertilização in vitro

Infertilidade

Técnicas reprodutivas assistidas

Hormônio liberador de

gonadotropina/agonistas

Keywords

Leuprolide/administration \& dosage

Fertilization in vitro

Infertility

Reproductive techniques, assisted

Gonadotropin-releasing hormone/agonists

\section{Uso de meia dose de agonista do GnRH de depósito para supressão hipofisária em ciclos de fertilização in vitro}

\author{
Half-dose long-acting form of GnRH agonist for pituitary \\ suppression in cycles of in vitro fertilization
}

\section{Resumo}

OBJETIVO: descrever a experiência de um serviço de reprodução assistida com a utilização de meia dose de agonista do GnRH de depósito para a supressão hipofisária em ciclos de fertilização in vitro (FIV). MÉTODOS: estudo prospectivo em que foram avaliados ciclos de FIV ou "intracytoplasmatic sperm injection" (ICSI) utilizando meia dose de acetato de leuprolide de depósito, iniciado na fase lútea média do ciclo menstrual, no período de agosto de 2005 a março de 2006. Foi administrado FSH recombinante para indução ovariana controlada em dose variada. O hCG era administrado quando pelo menos um folículo atingisse 19 mm de diâmetro máximo. Realizou-se FIV ou ICSI nos oócitos maduros de acordo com fator de infertilidade. Transferiram-se até quatro embriões por paciente no segundo ou terceiro dia após a captação. $\bigcirc$ uso de progesterona foi iniciado no mesmo dia da coleta oocitária. A dosagem sérica de $\beta$-hCG foi realizada no $14^{\circ}$ dia após a coleta dos oócitos. Foram avaliados os seguintes parâmetros: número de ciclos aspirados, ciclos cancelados e ciclos transferidos, quantidade total de FSH utilizado, número de oócitos maduros, taxa de fertilização, número de embriões transferidos, taxa de implantação embrionária e taxa de gestação clínica. RESULTADOS: 109 ciclos de FIV/ICSI utilizaram o protocolo descrito. A média de idade das pacientes foi 34,9 anos. A taxa de cancelamento foi de 1,8\% dos ciclos iniciados. Foram utilizadas $1.905 \mathrm{UI}$ de gonadotrofina, em média, por ciclo iniciado. Um total de $86,5 \%$ dos oócitos obtidos eram maduros, e a taxa de fertilização foi de 76,3\%. A média de embriões transferidos foi 2,7. As taxas de gestação por aspiração e por transferência foram 25,2 e 25,7\%, respectivamente. Um total de 26,3\% das gestações eram gemelares e 5,3\%, trigemelares. CONCLUSÃO: a administração de meia dose (1,87 mg) de acetato de leuprolide de depósito para bloqueio hipofisário pode ser utilizada com sucesso em ciclos de estimulação ovariana para FIV. Maior conforto, praticidade e menor custo são suas principais vantagens.

\section{Abstract}

PURPOSE: to evaluate the experience of an assisted reproduction center that uses depot administration of half-dose of $\mathrm{GnRH}$ agonist for pituitary suppression in assisted reproductive cycles. METHODS: prospective study that evaluated in vitro fertilization or intracytoplasmatic sperm injection (IVF/ICSI) cycles utilizing half-dose of leuprolide acetate between August 2005 and March 2006. Recombinant FSH was administered for controlled ovarian induction based on the protocol. hCG was administered when at least one follicle reached $19 \mathrm{~mm}$ in diameter. IVF or ICSI was performed according to infertility factor. Up to four embryos were transferred on the second or third day after oocyte retrieval. Progesterone supplementation was initiated on the same day of oocyte retrieval, and after 14 days $\beta$-hCG was measured. The following parameters were evaluated: number of aspirated cycles, cancelled cycles, transferred cycles, total dose of FSH employed, number of mature oocytes retrieved, fertilization rate, number of transferred embryos, embryo implantation rate, and pregnancy rate. RESULTS: A hundred and nine IVF/ICSI cycles were initiated. The mean age of the patients was 34.9 years. We observed $1.8 \%$ of cancellation rate. The mean total dose of gonadotrophins employed was 1,905 IU per cycle. We obtained $86.5 \%$ of mature oocytes and the fertilization rate was $76.3 \%$. The mean number of embryos transferred was 2.7. Pregnancy rates per aspiration and per transfer were 25.2 and $25.7 \%$, respectively. Of those who reached pregnancy, $26.3 \%$ were twins and $5.3 \%$ were triplets. CONCLUSIONS: the half-dose of GnRH depot employed for pituitary suppression was a useful alternative for ovarian stimulation in IVF cycles because it is comfortable and practical for the patient, besides its low cost.
Correspondência:

Évio Tognotti Avenida Angélica, 688, conj. 206 CEP $01228-000$ - São Paulo/SP Fone: (11) 3668-5771 E-mail: elviotognotiliglobo.com

Recebido $05 / 10 / 2006$

Aceito com modificacoũes 20/04/2007

\section{Projeto ALFA - Laboratório de Fertilização Assistida}

Projeto BETA - Clínica de Medicina Reprodutiva com Responsabilidade Social

- Diretor Clínico do Projeto BETA e Médico Assistente do Setor de Infertilidade da Clínica Ginecológica do Hospital das Clínicas da Faculdade de Medicina da Universidade de São Paulo - USP - São Paulo (SP), Brasil.

2Médico Assistente do Projeto BETA - São Paulo (SP), Brasil.

${ }_{3}^{3}$ Médico Assistente do Projeto BETA e Pós-graduando do Setor de Reprodução Humana do Departamento de Ginecologia da Escola Paulista de Medicina da Universidade Federal de São Paulo - UNIFESP/EPM - São Paulo (SP), Brasil.

${ }^{4}$ Médico Assistente do Projeto BETA e Diretor do Laboratório do Projeto ALFA.

${ }^{5}$ Diretor do Projeto BETA e Professor Assistente do Departamento de Ginecologia e Obstetrícia da Faculdade de Ciências Médicas da Santa Casa de São Paulo - São Paulo (SP), Brasil.

${ }^{\circ}$ Médico Assistente do Projeto BETA e Chefe do Setor de Infertilidade da Faculdade de Medicina do ABC - Santo André (SP), Brasil. 


\section{Introdução}

Os agonistas do hormônio liberador das gonadotropinas $(\mathrm{GnRH})$ têm sido largamente utilizados para supressão hipofisária em mulheres submetidas a estímulo ovariano controlado para ciclos de fertilização in vitro (FIV). Isto acontece porque apresentam vários benefícios, tais como: redução nas taxas de cancelamento, prevenção da luteinização precoce e do surgimento de pico de LH indesejado, melhora do recrutamento folicular, recuperação de maior número de oócitos e altas taxas de gestação ${ }^{1-5}$.

Os agonistas do GnRH de depósito (longa duração) foram primariamente desenvolvidos para uso em tratamentos de endometriose ou miomatose uterina e objetivam proporcionar níveis persistentes e prolongados de supressão hipofisária e conseqüente hipoestrogenismo. Tradicionalmente, os agonistas de curta duração em doses diárias são os mais utilizados em ciclos de FIV na maioria dos serviços, devido à preocupação de que a supressão hipofisária mais intensa obtida com a utilização das apresentações de depósito poderia causar alterações na fase lútea do ciclo, afetando negativamente as taxas de gestação e aumentando as taxas de abortamentos ${ }^{6}$. Além disso, uma intensa supressão hipofisária poderia ocasionar resposta ovariana mais lenta e gerar necessidade de maiores quantidades de gonadotropinas ${ }^{7,8}$, especialmente em pacientes com idade maior que 39 anos ${ }^{9}$. Entretanto, os agonistas de longa duração são bem aceitos por apresentarem administração mais simples e confortável (dose única) e conveniente (preço mais acessível).

Em ciclos de estímulo ovariano controlado, alguns autores acreditam que doses diárias menores de agonistas do GnRH sejam suficientes para supressão hipofisária e, a partir de estudos publicados, sugerem protocolos com a utilização de doses reduzidas, nos quais seriam necessárias menores quantidades de gonadotropinas sem, contudo, alteração nas taxas de sucesso $^{8-15}$. Outros autores, em número bem reduzido de trabalhos, avaliaram o uso de doses menores dos compostos de depósito. Eles não evidenciaram diferença significativa quanto à eficácia em dessensibilizar a hipófise, bem como não foram observadas alterações dos resultados nos ciclos de FIV $^{16,17}$. São raros os estudos que utilizaram menores doses de análogo do GnRH de depósito.

O objetivo deste estudo é apresentar dados, avaliar resultados e analisar a viabilidade da utilização de reduzidas doses de agonistas do GnRH de depósito para supressão hipofisária em ciclos de FIV.

\section{Métodos}

Foi realizado, no Projeto BETA, estudo prospectivo no período compreendido de $1^{\circ}$ de agosto de 2005 a 31 de março de 2006, com pacientes submetidas ao primeiro ciclo de estímulo ovariano controlado para FIV ou "intracytoplasmatic sperm injection" (ICSI). Todas as pacientes foram informadas do presente protocolo e consentiram em participar do estudo.

Foram considerados critérios de inclusão neste estudo: pacientes de 23 a 45 anos, com índice de massa corporal (IMC) compreendido entre $18-28 \mathrm{~kg} / \mathrm{m}^{2}$, infertilidade de causa feminina (tubária, ovulatória, endometriose), masculina ou idiopática e que não haviam utilizado qualquer droga dessensibilizadora da hipófise nos três meses anteriores ao início do estímulo ovariano para FIV ou ICSI. Após definição do grupo de estudo, o casal seria excluído caso desistisse do tratamento.

A média de idade das pacientes elegíveis foi de 34,9 anos, com variação de 23 a 44 anos; 79,8\% dos casais apresentavam infertilidade primária e 20,2\%, infertilidade secundária. Foram iniciados 109 ciclos de estimulação ovariana controlada. As causas de infertilidade destes casais eram: 47 casos por fator masculino, 27 por fator tubário, sete por endometriose, oito casos foram classificados como infertilidade sem causa aparente e em 20 ciclos houve mais de um fator responsável pela infertilidade conjugal.

Após realização de ultra-sonografia por via transvaginal, foram submetidas à supressão hipofisária na fase lútea do ciclo anterior $\left(18^{\circ}\right.$ ao $24^{\circ}$ dia do ciclo) com a utilização de $1,87 \mathrm{mg}$ de acetato de leuprolide de depósito por via subcutânea (Lupron ${ }^{\circledR}$ Depot, Abbott Laboratories, Chicago, EUA). As pacientes foram agendadas aos pares e a medicação diluída de maneira tradicional em $2 \mathrm{~mL}$; cada paciente recebia $1 \mathrm{~mL}$ do preparado.

No início do ciclo menstrual subseqüente (terceiro ao quinto dia) novo exame ultra-sonográfico foi realizado para confirmação do bloqueio ovariano. Iniciou-se o estímulo ovariano controlado nas pacientes que apresentavam imagem endometrial com espessura inferior a $5 \mathrm{~mm}$ e ovários sem dominância folicular (nenhum folículo maior que $10 \mathrm{~mm}$ ) com a utilização de folitropina beta (Puregon ${ }^{\circledR}$, Organon, Holanda) por via subcutânea, com dosagens que variaram de acordo com o seguinte protocolo: (i) $100 \mathrm{UI} /$ dia para pacientes com síndrome de ovários policísticos de acordo com o critério diagnóstico da ESHRE/ASRM ${ }^{18}$, (ii) $150 \mathrm{UI} /$ dia para pacientes com idade até 34 anos, (iii) 200 UI/ dia para pacientes com idade entre 35 e 39 anos, (iv) $300 \mathrm{UI} /$ dia para pacientes com 40 ou mais anos 
de idade. Após cinco dias, as doses eram ajustadas de acordo com as respostas individuais, aferidas por meio de ultra-sonografia transvaginal realizada no quinto e oitavo dias do estímulo.

Foi administrada a dose de 5.000 UI de hCG (Choriomon ${ }^{\circledR}$, Meizler Biopharma, Brasil) por via subcutânea quando pelo menos um folículo apresentasse diâmetro máximo de $19 \mathrm{~mm}$. Os níveis séricos de estradiol só foram dosados, quando necessário, para prevenção de síndrome de hiperestímulo ovariano. Os ciclos com nenhum folículo ovariano com pelo menos $14 \mathrm{~mm}$ no oitavo dia de estímulo foram cancelados por ausência de resposta. A aspiração folicular por via transvaginal guiada por ultra-sonografia foi realizada 35 horas após a administração do hCG.

Os tubos contendo $1 \mathrm{~mL}$ de HTF modificado mais o líquido folicular foram encaminhados imediatamente após a aspiração ao laboratório de manipulação de gametas. O líquido foi mantido em fluxo laminar horizontal sobre placa aquecida a $37^{\circ} \mathrm{C}$ até a sua manipulação; para identificação dos oócitos, o líquido folicular foi distribuído em placas pré-aquecidas e posteriormente observado à lupa estereoscópica em um aumento de 80 a 160X, neste momento sem meio de cultura. Após, os oócitos foram lavados em HTF modificado com $5 \%$ de substituto sintético de soro (SSS) e incubados até o momento da inseminação ou injeção em HTF com $10 \%$ SSS coberto com óleo. Para inseminação, o complexo cumulus-oophorus (CCO) devia apresentar características de oócito maduro e a qualidade espermática deveria ser adequada. Para injeção, os oócitos foram incubados por três horas e depois desnudados enzimática e mecanicamente, e classificados quanto a sua maturidade em microscópio invertido.

Foram selecionados três oócitos maduros (em metáfase II) para pacientes com idade até 34 anos, e quatro oócitos maduros para aquelas com 35 anos ou mais. Estes oócitos foram inseminados pela técnica FIV ou ICSI, conforme a avaliação seminal de cada caso. Nos procedimentos de FIV, após intervalo de três horas da incubação do CCO, foram adicionados ao meio de cultivo (HTF com SSS 10\%) cerca de 60.000 espermatozóides móveis por oócito com o auxílio de pipeta e ponteira graduada. Nestes casos, os $\mathrm{CCO}$ estavam incubados a $37^{\circ} \mathrm{C}, 5,5 \% \mathrm{CO}_{2}$ e $90 \%$ de umidade. Cada placa continha de três a quatro oócitos em microgotas de $20 \mu \mathrm{l}$, de acordo com a idade da paciente (até três oócitos para pacientes até 34 anos e até quatro oócitos para aquelas acima de 34 anos). Nos procedimentos de ICSI, os CCO foram banhados após três horas de incubação em solução de hialuronidase (80 UI) por 30 segundos, lavados novamente em solução de flushing
(HTF modificado com SSS 5\%) e incubados por cerca de 20 minutos a uma hora a $37^{\circ} \mathrm{C}, 5,5 \%$ de $\mathrm{CO}_{2}$ e $90 \%$ de umidade. Estes CCO, após o período de incubação, passaram pelo processo de denudação mecânica com pipetas de 130-140 $\mu \mathrm{m}$ e foram classificados.

A fertilização foi observada 18 horas após a inseminação ou injeção oocitária e os oócitos foram transferidos para meio HTF suplementado com SSS $15 \%$.

Cerca de 42 a 44 horas após a inseminação ou injeção, foi verificada a clivagem dos pré-embriões e conseqüente desenvolvimento embrionário em microscópio invertido sob aumento de 200 a 400X. A qualidade morfológica dos pré-embriões foi classificada conforme os critérios estabelecidos por Veeck ${ }^{19}$.

Após classificação, os pré-embriões foram fotografados, movidos para placa de transferência contendo $1 \mathrm{~mL}$ de HTF com SSS a $15 \%$ sem óleo e incubados a $37^{\circ} \mathrm{C}, 5,5 \% \mathrm{CO}_{2}$ e $90 \%$ de umidade até a hora da transferência. A transferência embrionária por via transcervical guiada por ultra-sonografia utilizando cateter SET TDT (Laboratoire CCD, Paris, França) foi realizada no segundo ou terceiro dia após aspiração folicular. Foram transferidos, no máximo, três embriões para pacientes de até 34 anos ou até quatro embriões para aquelas com 35 anos ou mais. A suplementação da fase lútea foi iniciada na noite da aspiração folicular com a utilização de $600 \mathrm{mg} / \mathrm{dia}$ de progesterona natural micronizada por via vaginal, dividida em três aplicações diárias (Evocanil ${ }^{\circledR}$, Zodiac, Brasil). A dosagem sérica de $\beta$-hCG foi realizada 14 dias após a aspiração folicular e, quando positiva, caracterizou gravidez bioquímica. Foi considerada gestação clínica quando a ultra-sonografia transvaginal evidenciou saco gestacional intra-útero.

Foram avaliados os seguintes parâmetros: número de ciclos aspirados, número de ciclos cancelados, quantidade total de FSH utilizado, número de oócitos maduros, taxa de fertilização, classificação embrionária, número de embriões transferidos, taxa de implantação, taxa de gestação clínica e taxa de gestação múltipla.

\section{Resultados}

Foram estudados 109 ciclos que iniciaram os procedimentos para FIV ou ICSI no período de agosto de 2005 a março de 2006. Destes, em 48 ciclos a idade da mulher era inferior a 35 anos, em 42 ciclos a idade estava compreendida entre 35 e 39 anos e 11 meses e em 19 ciclos as mulheres tinham 40 anos ou mais de idade. Todas as pacientes receberam a mesma dose de acetato de leuprolide de depósito $(1,87 \mathrm{mg})$ durante a fase lútea. 
Tabela 1 - Número de pré-embriões transferidos, taxas de implantação e de gestação por aspiração e por transferência, de acordo com faixas etárias.

\begin{tabular}{|c|c|c|c|c|c|}
\hline Idade (anos) & Ciclos aspirados/transferidos & $\begin{array}{c}\text { Pré-embriões } \\
\text { transferidos (média) }\end{array}$ & Implantaç̃̃o (\%) & $\begin{array}{c}\text { Gestação por } \\
\text { aspiracão (n) (\%) }\end{array}$ & $\begin{array}{c}\text { Gestação por } \\
\text { transferência (\%) }\end{array}$ \\
\hline$<35$ & $46 / 45$ & 2,4 & $14 / 110(12,7)$ & $11(23,9)$ & 24,4 \\
\hline $35-39$ & $42 / 41$ & 3,0 & $11 / 124(8,8)$ & $9(21,4)$ & 21,9 \\
\hline$\geq 40$ & $19 / 19$ & 2,9 & $9 / 56(16,0)$ & $7(36,8)$ & 36,8 \\
\hline Total & $107 / 105$ & 2,7 & $27 / 290(9,3)$ & $27(25,2)$ & 25,7 \\
\hline
\end{tabular}

Tabela 2 - Número de sacos gestacionais implantados segundo o grupo etário.

\begin{tabular}{lccc}
\hline Grupo efário (anos) & Única $-\mathrm{n}(\%)$ & Gemelar $-\mathrm{n}(\%)$ & Trigemelar $-(\%)$ \\
\hline$<35$ & $9(75)$ & $1(12,5)$ & $1(12,5)$ \\
$35-39$ & $7(71,4)$ & $2(28,6)$ & 0 \\
$\geq 40$ & $5(50)$ & $2(50)$ & 0 \\
Total & $21(68,4)$ & $5(26,3)$ & $1(5,3)$ \\
\hline
\end{tabular}

Observamos que apenas dois ciclos $(1,8 \%)$ foram cancelados por ausência de resposta, o que possibilitou aspiração folicular em 107 dos ciclos iniciados; em ambos os ciclos cancelados as pacientes tinham menos de 35 anos de idade. Foram utilizadas, na média, 1.905 UI de FSH recombinante por ciclo iniciado. Dos 107 ciclos aspirados, houve transferência embrionária em 105 deles: não houve embrião para ser transferido em um caso no grupo de pacientes até 34 anos e em um caso no grupo de pacientes com idade compreendida entre 35 e 39 anos.

Nos ciclos nos quais foi realizada FIV $(46,2 \%$ do total), foram obtidos $86,7 \%$ de oócitos maduros e $75,5 \%$ de fertilização, média de 8,1 oócitos maduros por caso; quando a técnica utilizada foi ICSI $(53,8 \%$ dos ciclos), foram obtidos $86,4 \%$ de oócitos maduros, a taxa de fertilização foi de $77,1 \%$ e a média de oócitos maduros foi de 8,1 .

Foram obtidos $38,7 \%$ de embriões classe I, 30,8\% classe II, 19,2\% classe III, 5,5\% eram pré-embriões classe IV e 5,2\% foram classificados como classe V. Apenas $0,6 \%$ dos pré-embriões foram transferidos em estágio de dois pró-núcleos.

A média de embriões transferidos foi 2,7; observamos $25,2 \%$ de gestações por aspiração, $25,7 \%$ de gestações por transferência e $9,3 \%$ de taxa de implantação; as diferenças entre os grupos etários estudados podem ser observadas na Tabela 1.

Entre as gestações obtidas com os tratamentos, $26,3 \%$ foram gemelares e $5,3 \%$ foram gestações trigemelares (Tabela 2).

\section{Discussão}

Desde que Rabin e $\mathrm{McNeil}^{20}$ propuseram o uso dos agonistas do GnRH para bloqueio hipofisário, o protocolo longo, com a combinação de agonistas do $\mathrm{GnRH}$ de uso diário, durante duas a quatro semanas, e gonadotropinas tem sido o mais utilizado em ciclos de estímulo ovariano controlado para FIV. O emprego deste protocolo apresenta benefícios bem estabelecidos e melhores taxas de gestação clínica ${ }^{21,22}$.

Os tratamentos de reprodução assistida são estressantes e cansativos para os casais inférteis. Um dos principais inconvenientes é a administração diária de medicamentos injetáveis que ficam na maioria dos casos sob responsabilidade da própria paciente. Portanto, qualquer tentativa de simplificar o tratamento é sempre motivo de grande interesse. Neste sentido, a utilização de uma única dose de agonista do GnRH de depósito se apresenta como opção possível ${ }^{9}$.

A utilização dos agonistas de depósito em ciclos de FIV apresenta vantagens quanto à conveniência, custos e efeitos colaterais quando comparada aos tratamentos que utilizam drogas de uso diário, com resultados aparentemente semelhantes, no que se refere à taxa de gestação e de abortamento ${ }^{8,23}$; apenas seis estudos bem conduzidos compararam a eficiência da utilização diária versus o uso da medicação em forma de depósito e os dados disponíveis na literatura só permitem concluir que a utilização de agonistas de depósito pode causar bloqueio hipofisário mais intenso, o que gera necessidade de maior quantidade de gonadotropinas para o estímulo ovariano e conseqüente aumento dos custos envolvidos no tratamento. As taxas de gestação e abortamentos encontradas são estatisticamente semelhantes. Nenhuma outra conclusão em favor de um dos métodos pode ser extraída dos trabalhos até então publicados ${ }^{24}$.

Devido à presunção de que a mais intensa supressão hipofisária possa gerar maior necessidade de gonadotropinas, alguns estudos foram realizados com o intuito de testar o resultado da diminuição na dose do agonista de $\mathrm{GnRH}$ de uso diário ou de depósito ${ }^{12,14,16,17,25}$. Estes mostraram que a administração de menor quantidade de agonista do GnRH reduz o grau de supressão hipofisária, com manutenção da capacidade de evitar elevações precoces de LH. Outros autores verificaram que a interrupção da administração diária de $\mathrm{GnRH}$ antes da aspiração folicular, inclusive no início do estímulo ovariano com gonadotropinas, possibilita prevenção do aparecimento do pico precoce de $\mathrm{LH}$, bem como não interfere nas taxas de fertilização e gravidez ${ }^{15}$. 
Concordante com a literatura, o presente estudo demonstrou que a utilização de $1,87 \mathrm{mg}$ de acetato de leuprolide de depósito foi capaz de evitar o surgimento de pico precoce de LH e a luteinização folicular prematura, o que pode ser depreendido pela taxa de recuperação oocitária, adequada taxa de oócitos maduros e baixa incidência de oócitos degenerados ou pós-maduros. O presente estudo revelou, também, boas taxas de fertilização e de gestação clínica. Acreditamos que as taxas de implantação observadas neste estudo sejam semelhantes àquelas descritas em outros estudos da literatura ${ }^{8,26}$.

Dal Prato et al. ${ }^{27}$ compararam dois grupos de pacientes que utilizam doses diferentes de agonista de depósito $(1,87$ e 3,75 mg) previamente à estimulação ovariana controlada. Verificaram que pacientes que recebem menor dose de triptorelina necessitam de menor quantidade de gonadotropinas, bem como apresentam maior número de oócitos recuperados, maior incidência de oócitos maduros e melhor taxa de fertilização. Não observaram diferença significativa entre as taxas de implantação, gestação ou abortamento.

Por outro lado, estudo randomizado com a utilização de doses distintas de triptorelina (1,87 e 3,75 mg) previamente à estimulação ovariana para FIV não mostrou diferença significativa no número de oócitos captados ou embriões produzidos. Também não relatou diferença nas taxas de gestação ou na dose total de gonadotropinas utilizada em pacientes que receberam diferentes doses do agonista de depósito ${ }^{17}$.

Acredita-se que há necessidade de ação direta do LH em seus receptores específicos no endométrio para adequado crescimento endometrial e receptividade uterina $^{28}$. É possível que a expressão endometrial de receptores de estrogênios e progestógenos esteja alterada em ciclos de estímulo ovariano controlado que apresentem baixos níveis de LH em conseqüência da utilização de agonistas de $\mathrm{GnRH}^{29}$. Sugerimos que a diferença nos resultados observada entre os estudos possa residir no tipo de gonadotropina utilizada no estímulo ovariano: FSH versus gonadotropina de mulheres menopausadas (hMG). Sabe-se que a hMG possui atividade LH e essa administração exógena de LH pode ter balanceado os efeitos de diferentes graus de supressão hipofisária induzidos por distintas doses de triptorelina. Embora necessite de estudos específicos para confirmação, acreditamos que doses menores de agonistas de $\mathrm{GnRH}$ podem proporcionar menor interferência na receptividade endometrial, diminuindo a necessidade de suplementação de LH ou mesmo utilizar FSH de forma isolada para a estimulação ovariana.

Estamos certos da necessidade de complementação deste estudo, trabalho que já se encontra em andamento, com a inclusão de grupo controle para reforçar os resultados observados. Porém, estamos confiantes de que as amplas vantagens que este esquema pode proporcionar, mantendo resultados satisfatórios, justificam sua utilização em várias circunstâncias. Se doses ainda menores $(1 / 3$ ou $1 / 4$ de ampola) podem resultar em resultados semelhantes ou melhores, ainda deverá ser investigado.

Em conclusão, os resultados indicam que a administração de meia dose $(1,87 \mathrm{mg})$ de acetato de leuprolide de depósito por via subcutânea para bloqueio hipofisário pode ser utilizada com sucesso em ciclos de estimulação ovariana para FIV. Maior conforto, praticidade e menor custo são suas principais vantagens.

\section{Referências}

1. Caspi E, Ron-El R, Golan A, Nachum H, Herman A, Soffer $Y$, Weinraub Z. Results of in vitro fertilization and embryo transfer by combined long-acting gonadotropin-releasing hormone analog D-Trp-6-luteinizing hormone-releasing hormone and gonadotropins. Fertil Steril. 1989; $51(1): 95-9$

2. Liu HC, Lai YM, Davis O, Berkeley AS, Graf M, Grifo J, et al. Improved pregnancy outcome with gonadotropin releasing hormone agonist ( $\mathrm{GnRH}-\mathrm{a}$ ) stimulation is due to the improvement in oocyte quantity rather than quality. J Assist Reprod Genet. 1992; 9(4):338-44.

3. Hazout A, de Ziegler D, Cornel C, Fernandez H, Lelaidier C, Frydman R. Comparison of short 7-day and prolonged treatment with gonadotropin-releasing hormone agonist desensitization for controlled ovarian hyperstimulation. Fertil Steril. 1993; 59(3):596-600.
4. Testart J, Lefevre B, Gougeon A. Effects of gonadotrophin-releasing hormone agonists (GnRHa) on follicle and oocyte quality. Hum Reprod. 1993; 8(4):511-8.

5. Tapanainen J, Hovatta O, Juntunen K, Martikainen H, Ratsula K, Tulppala $M$, et al. Subcutaneous goserelin versus intranasal buserelin for pituitary down-regulation in patients undergoing IVF: a randomized comparative study. Hum Reprod. 1993; 8(12):2052-5.

6. Devreker F, Govaerts I, Bertrand E, Van den Bergh M, Gervy $C$, Englert $Y$. The long-acting gonadotropin-releasing hormone analogues impaired the implantation rate. Fertil Steril. 1996. 65(1):122-6

7. Ben-Rafael Z, Lipitz S, Bider D, Mashiach S. Ovarian hyporresponsiveness in combined gonadotropin-releasing hormone agonist and menotropin therapy is associated with low serum follicle-stimulating hormone levels. Fertil Steril. 1991; 55(2):272-5. 
8. Sonntag B, Kiesel L, Nieschlag E, Behre HM. Differences in serum LH and FSH levels using depot or daily GnRH agonists in controlled ovarian stimulation: influence on ovarian response and outcome of ART. J Assist Reprod Genet. 2005; 22(7-8):277-83.

9. Geber S, Sales L, Sampaio MA. Comparison between a single dose of goserelin (depot) and multiple daily doses of leuprolide acetate for pituitary suppression in IVF treatment: a clinical endocrinological study of the ovarian response. J Assist Reprod Genet. 2002; 19(7):313-8.

10. Feldberg D, Farhi J, Ashkenazi J, Dicker D, Shalev J, Ben-Rafael Z. Minidose gonadotropin-releasing hormone agonist is the treatment of choice in poor responders with high follicle-stimulating hormone levels. Fertil Steril. 1994; 62(2):343-6.

11. Olivennes F, Righini C, Fanchin R, Torrisi C, Hazout A, Glissant $M$, et al. A protocol using a low dose of gonadotrophin-releasing hormone agonist might be the best protocol for patients with high follicle-stimulating hormone concentrations on day 3 . Hum Reprod. 1996; $11(6): 1169-72$.

12. Janssens RM, Lambalk CB, Vermeiden JP, Schats R, Bernards JM, Rekers-Mombarg LT, et al. Dose-finding study of triptorelin acetate for prevention of a premature LH surge in IVF: a prospective, randomized, double-blind, placebo-controlled study. Hum Reprod. 2000; 15(1 1):2333-40.

13. Dal Prato L, Borini A, Trevisi MR, Bonu MA, Sereni E, Flamigni C. Effect of reduced dose of triptorelin at the start of ovarian stimulation on the outcome of IVF: a randomized study. Hum Reprod. 2001; 16(7): 1409-14.

14. Fabregues F, Penarrubia J, Creus M, Casamitjana R, Vanrell JA, Balasch J. Effect of halving the daily dose of triptorelin at the start of ovarian stimulation on hormone serum levels and the outcome of in vitro fertilization. Fertil Steril. 2005; 83(3):785-8.

15. Simons AH, Roelofs HJ, Schmoutziguer AP, Roozenburg BJ, van't Hof-van den Brink EP, Schoonderwoerd SA. Early cessation of triptorelin in in vitro fertilization: a double-blind, randomized study. Fertil Steril. 2005; 83(4):889-96.

16. Balasch J, Gomez F, Casamitjana R, Carmona F, Rivera F, Vanrell JA. Pituitary-ovarian suppression by the standard and half-doses of D-Trp-6-luteinizing hormone-releasing hormone depot. Hum Reprod. 1992; 7(9): 1230-4

17. Yim SF, Lok IH, Cheung LP, Briton-Jones CM, Chiu TT, Haines CJ. Dose-finding study for the use of long-acting gonadotrophinreleasing hormone analogues prior to ovarian stimulation for IVF. Hum Reprod. 2001; 16(3):492-4.

18. The Rotterdam ESHRE/ASRM-Sponsored PCOS consensus workshop group. Revised 2003 consensus on diagnostic criteria and long- term health risks related to polycystic ovary syndrome (PCOS). Hum Reprod. 2004; 19(1):41-7.

19. Veeck LL. Oocyte assessment and biological performance. Ann N Y Acad Sci. 1988; 541:259-74.

20. Rabin $D, M c N e i l$ LW. Pituitary and gonadal desensitization after continuous luteinizing hormone-releasing hormone infusion in normal females. J Clin Endocrinol Metab. 1980;51 (4):873-6.

21. Rutherford AJ, Subak-Sharpe RJ, Dawson KJ, Margara RA, Franks $S$, Winston RM. Improvement of in vitro fertilisation after treatment with buserelin, an agonist of luteinising hormone releasing hormone. Br Med J (Clin Res Ed). 1988; 296(6639): 1765-8.

22. Daya S. Long versus short gonadotropin releasing hormone agonist protocols for pituitary desensitization in assisted reproduction cycles. Oxford: Update Software; 1999. (The Cochrane Library, issue 3).

23. Gianaroli L, Ferraretti AP, Feliciani E, Tabanelli C, Magli C, Fortini D. Prospective randomized study of D-Trp6-LHRH versus buserelin in long desensitization protocols for medically assisted conception cycles. Hum Reprod. 1994; 9(2):220-5.

24. Albuquerque LE, Saconato H, Maciel MC, Baracat EC, Freitas V. Depot versus daily administration of $\mathrm{GnRH}$ agonist protocols for pituitary desensitization in assisted reproduction cycles: a Cochrane Review. Hum Reprod. 2003; 18(10):2008-17.

25. Hsieh Y, Tsai H, Chang C, Lo H. Comparison of a single half-dose, long-acting form of gonadotropin-releasing hormone analog $(\mathrm{GnRH}$ a) and a short-acting form of GnRH-a for pituitary suppression in a controlled ovarian hyperstimulation program. Fertil Steril. 2000; 73(4):817-20.

26. Registro Latinoamericano de Reproducción Asistida. Tasa de implantación de acuerdo a la edad de la mujer en FIV, ICSI y Hatching Asistido [homepage de la Internet]. 2003 [citado 12 abr 2007]. Disponible en: http://www.redlara. com/reg_2003.asp

27. Dal Prato L, Borini A, Coticchio G, Cattoli M, Flamigni C. Halfdose depot triptorelin in pituitary suppression for multiple ovarian stimulation in assisted reproduction technology: a randomized study. Hum Reprod. 2004; 19(10):2200-5.

28. Tesarik J, Hazout A, Mendoza C. Luteinizing hormone affects uterine receptivity independently of ovarian function. Reprod Biomed Online. 2003; 7(1):59-64.

29. Bourgain C, Ubaldi F, Tavaniotou A, Smitz J, Van Steirteghem AC, Devroey P. Endometrial hormone receptors and proliferation index in the periovulatory phase of stimulated embryo transfer cycles in comparison with natural cycles and relation to clinical pregnancy outcome. Fertil Steril. 2002; 78(2):237-44. 